\title{
Anaemia: Approach to diagnosis
}

\author{
N Alli, ${ }^{1} \mathrm{MB}$ BCh, FCPathHaem (SA); J Vaughan, ${ }^{1} \mathrm{MB}$ BCh, FCPathHaem (SA), MMed (Haem); \\ M Patel, ${ }^{2}$ MB ChB, FCP (SA), MMed, FRCP (Lond), PhD \\ ${ }^{1}$ Department of Molecular Medicine and Haematology, School of Pathology, Faculty of Health Sciences, University of the Witwatersrand, \\ Johannesburg, and National Health Laboratory Service, Johannesburg, South Africa \\ ${ }^{2}$ Department of Clinical Haematology, Division of Internal Medicine, Chris Hani Baragwanath Academic Hospital, Johannesburg, \\ and School of Medicine, Faculty of Health Sciences, University of the Witwatersrand, Johannesburg, South Africa
}

Corresponding author: N Alli (nazeer.alli@nhls.ac.za)

\begin{abstract}
Anaemia is defined as a condition in which the number of red cells or their oxygen-carrying capacity is insufficient to meet physiological needs. It is the most common disorder globally and one of the conditions that general practitioners most frequently encounter. In the World Health Organization global database, anaemia is estimated to affect 1.6 billion people. As anaemia manifests in a wide range of conditions, it is important to embrace a structured diagnostic approach. The recommended approach set out in this article incorporates clinical and pathophysiological considerations, red cell characteristics, and bone marrow activity. In this issue of CME, the first of two parts on anaemia, the causes of anaemia related specifically to decreased red cell production are discussed.
\end{abstract}

S Afr Med J 2017;107(1):23-27. DOI:10.7196/SAMJ.2017.v107i1.12148

Red cells are produced in the bone marrow (BM) from myeloid progenitor cells, where their production requires a permissive BM micro-environment and adequate substrate (including iron, vitamin $\mathrm{B}_{12}$ and folate) for $\mathrm{Hb}$, protein and DNA synthesis. Erythropoiesis is controlled by erythropoietin (EPO), which is synthesised by peritubular fibroblasts in the renal cortex in response to reduced oxygen tension.

According to the World Health Organization (WHO), anaemia is defined as 'a condition in which the number of red blood cells or their oxygen-carrying capacity is insufficient to meet physiologic needs'. It is the most common disorder globally and one of the conditions that general practitioners most frequently encounter. Anaemia is rarely an isolated disease and is most often a sign of an acquired or inherited disorder. ${ }^{[1]}$

According to the WHO global database, ${ }^{[2]}$ anaemia is estimated to affect 1.6 billion people. The highest prevalence is found in preschool-age children (47.4\%), followed by pregnant females (41.8\%), non-pregnant females (30.2\%), school-age children (25.4\%), and males $(12.7 \%){ }^{[2]}$

When investigating patients with anaemia, two fundamental questions have to be considered: (i) What is the cause of the anaemia?; and (ii) What is the urgency for correcting the anaemia, i.e. is a blood transfusion or other urgent intervention indicated?

\section{Classification}

Anaemia may be classified as follows:

Based on red cell characteristics (red cell size, chromia and morphology):

- hypochromic microcytic

- macrocytic normochromic

- normochromic normocytic

- morphology:

- leuco-erythroblastic

- micro-/macroangiopathic.

Based on underlying mechanism:

- decreased BM production/output:

- BM aplasia/infiltrate
- ineffective haematopoiesis, e.g. megaloblastic anaemia (MA), myelodysplastic syndromes (MDSs), HIV

- substrate deficiency

- EPO insufficiency

- peripheral loss/destruction:

- bleeding

- sequestration

- haemolysis.

In this issue of CME, the focus is on anaemia resulting from decreased BM output. Anaemia caused by peripheral loss will be discussed in the next issue.

\section{Diagnostic approach to a patient with suspected anaemia}

As anaemia may manifest in a wide range of disorders, a streamlined diagnostic approach is vital (Fig. 1). The approach outlined here is based on the abovementioned classification, clinical scenario and full blood count (FBC) findings. ${ }^{[3]}$

Patients generally present with symptoms of anaemia, i.e. increased tiredness/fatigue, dyspnoea and decreased effort tolerance. The severity of symptoms depends on the degree of anaemia and rate of $\mathrm{Hb}$ decrease. Therefore, at a given $\mathrm{Hb}$ level, anaemia from acute blood loss is likely to manifest more severely than anaemia of insidious onset (weeks to months). Symptoms during early childhood should remind one of possible inherited forms of anaemia, e.g. thalassaemia.

\section{History}

A detailed history is of paramount importance and often eliminates much of the speculation during investigation. This should include:

- interrogation of the presenting complaint and duration of the problem

- transfusion history

- dietary history, including pica (craving for unusual food items, generally associated with iron deficiency)

- travel history (to endemic malarial or other infectious areas)

- change in bowel habits

- bleeding (e.g. gastrointestinal and genito-urinary) 


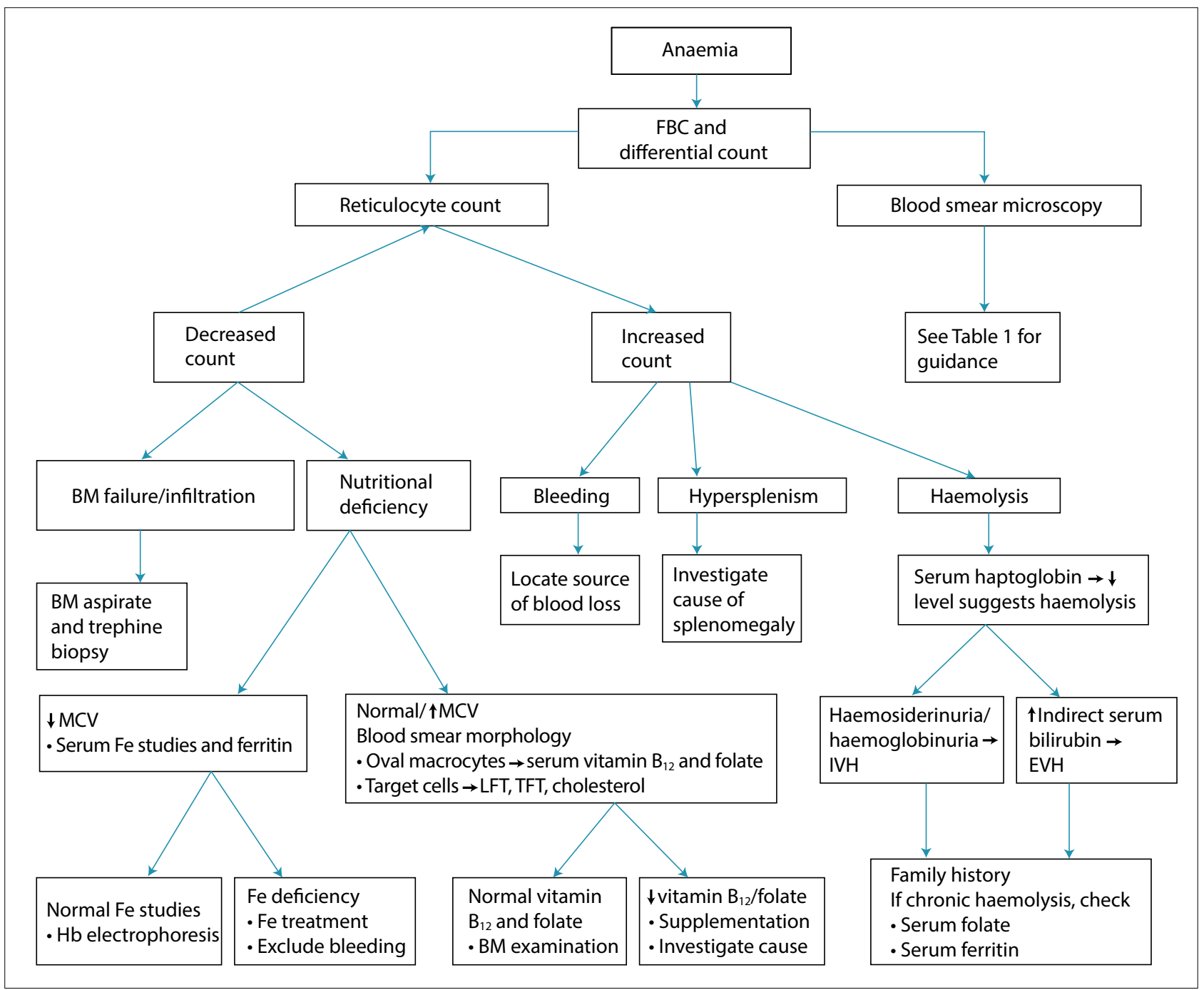

Fig. 1. Algorithm depicting diagnostic approach to anaemia. $(T F T=$ thyroid function test; $L F T=$ liver function test $M C V=$ mean cell volume; $I V H=$ intravascular haemolysis; $E V H=$ extravascular haemolysis; $F B C=$ full blood count; $F e=$ iron. $)$

- drug history (e.g. anticoagulants, antiplatelet agents, renotoxic agents, anticonvulsants)

- chronic disease (e.g. HIV, tuberculosis (TB))

- surgery (e.g. gastrectomy, small-bowel surgery)

- current or recent pregnancy

- family history (particularly in children).

\section{Clinical examination}

A wide range of signs and symptoms may be apparent. Systematic examination directs further investigation and may reveal the possible cause.

- Skin and mucous membrane:

- pallor is the cardinal clinical sign for anaemia, which should be confirmed by measuring the $\mathrm{Hb}$ level

- angular stomatitis

- glossitis in nutritional deficiencies

- koilonychia (spoon-shaped nails) in iron deficiency

- premature greying, which often accompanies MA
- scleral ictus, which indicates possible haemolysis or ineffective erythropoiesis.

- Neuromuscular:

- muscle weakness

- headache, lack of concentration, drowsiness, tinnitus

- paraesthesias, peripheral neuropathy, ataxia and loss of vibration sense, and proprioception in pernicious anaemia.

- Cardiovascular:

- hyperdynamic circulation with haemic 'flow' murmurs

- cardiac failure.

- Clues for infection, malignancy (e.g. lymphoma, leukaemia, metastatic carcinoma):

- hepatosplenomegaly

- lymphadenopathy

- bleeding manifestations (petechiae, purpura, ecchymosis), BM failure.

\section{Laboratory testing}

An FBC, differential and reticulocyte counts together with microscopic blood smear examination should be the starting point of investigations. These confirm the clinical suspicion of anaemia and direct further investigation. Local laboratory/population reference ranges that are age and gender specific should be used. The normal reference ranges for $\mathrm{Hb}$ in adults in the Witwatersrand area, Johannesburg, South Africa (SA) are as follows: ${ }^{[4]}$

- male: 13.8 - $17.9 \mathrm{~g} / \mathrm{dL}$ (anaemia $<13 \mathrm{~g} / \mathrm{dL}$ )

- female: 12.4 - $15.5 \mathrm{~g} / \mathrm{dL}$ (anaemia $<12 \mathrm{~g} / \mathrm{dL}$; pregnancy $<11 \mathrm{~g} / \mathrm{dL}$ ).

A reticulocyte count gives an indication of the BM status, i.e. decreased activity v. appropriate response to the anaemia. A reticulocyte production index (RPI) provides a more accurate representation of marrow activity than an isolated reticulocyte count, as it corrects for the degree of anaemia and presence of immature reticulocytes in the peripheral blood. The RPI is calculated as follows: 
Table 1. Red cell morphological characteristics

\begin{tabular}{|c|c|c|}
\hline Morphological observation & Significance & Further tests indicated and expected result \\
\hline $\begin{array}{l}\text { Oval macrocytes, teardrops, } \\
\text { basophilic stippling, right shift }\end{array}$ & MA & Serum vitamin $B_{12}$ and folate levels \\
\hline Hypochromia & Iron deficiency anaemia (also see pencil cells) & Serum ferririn, transferrin and iron levels \\
\hline Microcytosis & Chronic disorder; thalassaemia trait & Hb electrophoresis/HPLC \\
\hline Sickle cells & Seen in sickle cell disease & $\begin{array}{l}\mathrm{Hb} \text { electrophoresis to confirm presence of } \mathrm{Hb} \mathrm{S} \text {, } \\
\text { and quantitate } \mathrm{Hb} F \text { level }\end{array}$ \\
\hline Spherocytes & Noted in hereditary spherocytosis, warm AIHA & $\begin{array}{l}\text { Coombs test: positive for IgG in warm AIHA } \\
\text { Red cell membrane analysis: selected membrane } \\
\text { protein abnormalities }\end{array}$ \\
\hline Elliptocytes/ovalocytes & Hereditary elliptocytosis/ovalocytosis & $\begin{array}{l}\text { Red cell membrane analysis: selected membrane } \\
\text { protein abnormalities }\end{array}$ \\
\hline Autoagglutination & Cold AIHA & Coombs test: positive for C $3 \mathrm{~d}$ in cold AIHA \\
\hline Red cell fragmentation & $\begin{array}{l}\text { If platelets decreased } \rightarrow \text { microangiopathic } \\
\text { haemolysis } \\
\text { If platelets normal } \rightarrow \text { macroangiopathic haemolysis }\end{array}$ & $\begin{array}{l}\text { Microangiopathic haemolysis: DIC screen } \rightarrow \\
\text { consumptive coagulopathy U\&E: marked renal } \\
\text { dysfunction in HUS } \\
\text { Altered neurological status: suspect TTP }\end{array}$ \\
\hline Malaria & Life-threatening infection & $\begin{array}{l}\text { Identify species } \\
\text { Monitor FBC and parasite count while on treatment }\end{array}$ \\
\hline
\end{tabular}

- $\%$ reticulocytes $\times$ patient haematocrit $/ 45 \div$ reticulocyte maturation time (days) in peripheral blood

The reticulocyte maturation time is calculated as follows:

- haematocrit $>40 \%=1$ day, $30-40 \%=1.5$ days, $20-30 \%=2$ days, $<20 \%=2.5$ days

A decreased RPI signifies a suboptimal BM response for correction of the anaemia. Clues for further investigation are derived from red cell indices (Fig. 1) and peripheral blood smear microscopy (Table 1).

$\mathrm{BM}$ examination is appropriate if pathology is suspected, e.g. BM infiltration, BM failure, and myelodysplasia. BM aspirate smears permit morphological assessment in cellular detail, but the architecture is disrupted, whereas in trephine biopsy sections the architecture is preserved, which makes it possible to detect pathology, such as granulomata and fibrosis.

More specialised tests are performed in individuals suspected of having rarer conditions that cause anaemia.

\section{Important causes of anaemia due to inadequate $\mathrm{BM}$ output}

Causes of red cell production failure include EPO deficiency (usually secondary to chronic kidney disease), substrate deficiency, ineffective erythropoiesis, BM aplasia/hypoplasia and BM displacement (malignancy/fibrosis), all of which are characterised by a low RPI.

\section{BM aplasia/hypoplasia (primary BM failure)}

BM failure may be inherited or acquired. In SA, the majority of patients have acquired idiopathic aplastic anaemia (AA). Inherited BM failure syndromes generally manifest in childhood and represent $<10 \%$ of patients with AA.
Patients may present with one or more of the following: $(i)$ symptoms of anaemia; (ii) infection (due to leucopenia/neutropenia); and (iii) mucocutaneous bleeding (due to thrombocytopenia). The FBC shows a pancytopenia of varying degrees (depending on the severity), reticulocytopenia with a low RPI, and reversal of the normal neutrophil:lymphocyte ratio. The diagnosis is confirmed on microscopic BM examination.

Numerous secondary causes and associations have been identified in $\mathrm{AA}$, but in $60-70 \%$ of cases the cause remains unknown. It has been proposed that a triggering event, such as a virus or drug, leads to an immune response and to inappropriate expansion of cytotoxic $\mathrm{T}$ cells that destroy haematopoietic cells. Causes include viruses (e.g. hepatitis virus, HIV, Epstein-Barr virus, cytomegalovirus); autoimmune disorders (e.g. systemic lupus erythematosis, eosinophilic fasciitis, Sjögren's syndrome); drugs (a wide range, including sulfonamides, anticonvulsants, antimalarials; chemicals (e.g. benzene); and environmental factors (e.g. agricultural pesticides and radiation). The idiopathic variety is the largest group. Paroxysmal nocturnal haemoglobinuria (PNH), caused by expansion of a clone with mutation of the glycosylphosphatidyl-inositol gene, manifests with the clinical triad of haemolysis, thrombosis and BM failure. PNH clones can be detected on flow cytometry.

Some inherited disorders are characterised by BM hypoplasia/ aplasia (affecting one or more haematopoietic cell lines), and are usually accompanied by one or more somatic abnormalities, i.e. part of a clinical syndrome. Inherited BM failure syndromes may be present at birth or develop later in childhood. Of these, the syndromes that affect all three cell lines include Fanconi anaemia, SchwachmanDiamond syndrome and dyskeratosis congenita. Further discussion of these syndromes is beyond the scope of this edition of CME.

Pure red cell aplasia is characterised by an isolated, often severe anaemia with a marked reticulocytopenia. It may be congenital 
(Diamond Blackfan syndrome) or acquired (including viral infections, lymphoproliferative disorders, autoimmune disorders or drugs). In SA, parvovirus B19 infection in HIV-positive individuals is the most common cause of pure red cell aplasia. BM examination reveals the absence or maturation block of erythroid progenitors.

Patients with BM failure should be referred to a specialist haematology/oncology unit for further work-up and management.

\section{BM displacement by malignancy or fibrosis}

The presence of a leuco-erythroblastic reaction and teardrop poikilocytosis on peripheral blood smear microscopy should lead one to suspect possible BM infiltration.

Leukaemia and lymphoma can be further characterised on flow cytometry, cytogenetic analysis and polymerase chain reaction testing on a bone marrow aspirate sample. Immunohistochemical stains are also available to identify subtypes and cell types on BM trephine biopsy sections.

\section{Ineffective haematopoiesis}

In ineffective haematopoiesis, the BM activity ranges from normal to increased, but cells die before or shortly after release into the circulation. Common causes for ineffective haematopoiesis include MA (discussed below), drugs that interrupt DNA synthesis or folate metabolism (e.g. methotrexate, trimethoprim, phenytoin), HIV infection, and the MDSs. The MDSs are clonal haematopoietic disorders characterised by peripheral blood cytopenias and dysplastic haematopoiesis, with a predisposition towards developing acute leukaemia.

When suspected, MA should be excluded by means of serum vitamin $B_{12}$ and folate levels (performed prior to blood transfusion), as should HIV and other chronic infections. Where no other cause for ineffective haematopoiesis is found, further work-up for MDSs may be necessary (including BM examination with cytogenetic analysis), particularly in older patients.

\section{Substrate deficiency}

Iron deficiency is the most common cause of anaemia, occurring in an estimated $15 \%$ of the world's population. ${ }^{[5]}$ The causes of iron deficiency can be broadly classified as inadequate iron intake (e.g. nutritional deficiency, iron malabsorption) or excessive iron loss (mostly due to bleeding). It is most common in young children and women of childbearing age because of the increased iron demands related to rapid growth and menstrual blood losses/pregnancy, respectively.

Iron deficiency causes a microcytic and/or hypochromic anaemia. Serum iron studies show low serum ferritin and raised transferrin (TF) levels, and low $\mathrm{TF}$ percentage saturation. The differential diagnosis for microcytic anaemia includes anaemia of chronic disorder (ACD), thalassaemia trait and sideroblastic anaemia. The frequency of these differential diagnoses depends on the demographics of the population served, with thalassaemia trait being common in patients of Indian or Mediterranean ancestry, and ACD occurring frequently in patients with chronic infection (e.g. HIV or TB), malignancy or autoimmune disorders. ACD is caused by a combination of functional iron deficiency (due to reticuloendothelial iron blockade), and EPO deficiency or resistance, and is characterised on iron studies by raised ferritin and low TF levels with low or normal TF percentage saturation. The anaemia is usually normocytic and normochromic, but can be microcytic in approximately one-third of cases due to long-standing iron restriction at the macrophage level.
Occasionally, the distinction between iron deficiency anaemia (IDA) and ACD can be blurred, as ferritin is an acute-phase protein and may be falsely normal or raised in patients with iron deficiency in the presence of acute inflammation. In this setting, the gold standard for discriminating IDA from ACD is evaluating BM iron stores microscopically. Measuring the soluble TF receptor (STFR) levels and calculating the STFR index (STFR:log ferritin ratio) is also of use in this regard, where a ratio $>2$ suggests iron deficiency with coexisting ACD ${ }^{[6]}$ Unfortunately, STFR testing is not universally available in SA laboratories. Of note is that serum iron levels are of minimal use in assessing iron stores, reflecting recent iron intake as opposed to overall iron status. IDA should therefore not be diagnosed solely on the basis of a low serum iron level.

To establish if someone has iron deficiency, one has to include a thorough dietary history, with emphasis on the frequency of red meat consumption, excessive dietary phytates (present in grains and legumes) or tannins (present in tea), which inhibit iron absorption, and pica. In the absence of an obvious nutritional cause, a source of bleeding should be sought (e.g. urogenital, gastrointestinal), particularly in men and postmenopausal women.

Oral iron supplementation is the mainstay of therapy for IDA, but intravenous iron is occasionally necessary when more rapid correction of the $\mathrm{Hb}$ level is desired, if iron malabsoprtion is the main cause for the IDA, or if oral iron is not tolerated. A blood transfusion is indicated for severe, symptomatic anaemia, especially in patients who are bleeding. Treatment of IDA should aim at restoring the $\mathrm{Hb}$ to normal, followed by a further 4 - 6 months of therapy to replenish iron stores (Table 2). Iron should ideally be given between meals together with vitamin $C$ to maximise absorption, but it can be administered with food or at reduced doses in patients who experience excessive abdominal side-effects (e.g. abdominal discomfort, nausea or constipation). For patients who are refractory to oral iron therapy, iron malabsorption, ongoing blood loss and non-compliance should be considered.

The treatment of ACD is centred on management of the underlying cause. There may be a role for EPO supplementation, particularly in patients with underlying malignancy ${ }^{[7]}$ and HIV.$^{[8]}$ Intravenous iron may additionally benefit patients with functional iron deficiency. ${ }^{[9]}$

MA is the other major nutritional anaemia caused by vitamin $B_{12}$ or folate deficiency. As both are required for purine biosynthesis, a deficiency of either nutrient hampers normal DNA synthesis, which results in ineffective haematopoiesis.

Vitamin $B_{12}$ deficiency is the most frequent cause of MA seen in general practice, the most common cause being pernicious

Table 2. Suggested therapeutic protocols for nutritional anaemia

\begin{tabular}{|c|c|}
\hline Micronutrient deficiency & Suggested therapeutic protocol \\
\hline \multirow[t]{4}{*}{ Iron } & Ferrous sulphate $200 \mathrm{mg}$ orally \\
\hline & 3 times daily between meals \\
\hline & together with vitamin $\mathrm{C}$ for \\
\hline & $4-6$ months \\
\hline \multirow{3}{*}{ Folate } & $5 \mathrm{mg}$ orally daily for 2 - \\
\hline & 4 months (or while the \\
\hline & underlying risk factor persists) \\
\hline \multirow[t]{6}{*}{ Vitamin $\mathrm{B}_{12}$} & $1000 \mu \mathrm{g}$ daily intramuscularly \\
\hline & for 5 - 7 days, followed by \\
\hline & $1000 \mu \mathrm{g}$ intramuscularly \\
\hline & weekly for a month, and then \\
\hline & $1000 \mu \mathrm{g}$ intramuscularly every \\
\hline & 2 months for life \\
\hline
\end{tabular}


Table 3. Causes of vitamin $B_{12}$ and folate deficiencies

\begin{tabular}{ll}
\hline Vitamin $\mathbf{B}_{\mathbf{1 2}}$ deficiency & Folate deficiency \\
\hline Dietary deficiency & Dietary deficiency \\
Ovolactovegetarians & Poor diet (e.g. alcoholics, elderly) \\
Malabsorption & Malabsorption \\
Pernicious anaemia & Inflammatory bowel disease \\
Ileal pathology & Coeliac disease \\
Crohn's disease/ulcerative colitis & Short-bowel syndrome \\
Infection, e.g. HIV, TB & Impaired folate metabolism \\
Infiltration, e.g. lymphoma surgery & Drugs (e.g. methotrexate, anticonvulsants, antimalarials) \\
Gastrectomy, ileal resection & Alcoholism \\
Diphyllobothrium latum (fish tapeworm) & Hypothyroidism \\
Blind loop syndrome & Excessive folate demand \\
Drugs (e.g. proton pump inhibitors, metformin) & Chronic haemolytic anaemia \\
& Pregnancy and lactation (particularly if the diet is poor)
\end{tabular}

anaemia. The latter leads to impaired vitamin $B_{12}$ absorption owing to immune-mediated destruction of the gastric parietal cells and consequent intrinsic factor deficiency. In contrast, dietary deficiency and the postpartum phase most commonly cause folate depletion. Dietary deficiency is currently relatively uncommon in SA owing to mandatory fortification of maize meal and wheat flour. Other causes of vitamin $B_{12}$ and folate deficiency are summarised in Table 3 .

MA is diagnosed by demonstrating low serum levels of vitamin $B_{12}$ or folate, along with characteristic peripheral blood smear morphology (including macro-ovalocytosis, teardrops, varying numbers of red cell fragments and basophilic stippling, and hypersegmentation (right shift) of neutrophils). Importantly, MA is not universally macrocytic, and cannot be excluded solely owing to the presence of a normal or even a low mean cell volume. When MA is strongly suspected, a trial of vitamin $B_{12}$ and folate supplementation is advisable, particularly if levels are borderline low.

When vitamin $B_{12}$ deficiency is diagnosed, further investigation for pernicious anaemia is warranted. Intrinsic-factor antibodies are positive in $50-70 \%$ of cases. ${ }^{[10]}$ In patients with pernicious anaemia, other organ-specific auto-immune disorders should be excluded (e.g. Addison's disease and auto-immune thyroiditis). Vitamin $B_{12}$ deficiency is treated with lifelong parenteral vitamin $B_{12}$ replacement therapy, while folate depletion is treated with oral folate supplementation (Table 3). Importantly, folate administration in patients with MA secondary to vitamin $B_{12}$ deficiency may partially correct the anaemia, but does not address the effects of vitamin $B_{12}$ deficiency on the central nervous system. Owing to the risk of neurological complications (such as subacute combined degeneration of the spinal cord) in patients with vitamin $B_{12}$ deficiency, MA should not be presumptively treated with folate alone, as this may accelerate irreversible neurological damage. Although the mainstay of treatment in vitamin $B_{12}$ deficiency is supplementation with vitamin $B_{12}$, patients with very severe/life-threatening anaemia may benefit from a blood transfusion. Crucially, these patients should be transfused conservatively and cautiously (no more than 1 or 2 units of blood should be transfused, each unit running over $\sim 2$ - 4 hours, followed by $20 \mathrm{mg}$ of intravenous furosemide (Lasix) after each unit), as aggressive transfusion practices are associated with a risk of sudden death in this setting.

\section{Conclusion}

There are numerous and varied causes of anaemia. Given the vastness of the topic, a thorough history, physical examination and systematic laboratory investigation are key elements towards determining the cause of the condition.

1. World Health Organization. The Global Prevalence of Anaemia in 2011. Geneva: WHO, 2015 ,

2. De Benoist B, McLean E, Ines E, et al. Worldwide prevalence of anaemia 1993 - 2005. Geneva: WHO 2008

3. Means RT Jr, Glader B. Anaemia: General considerations. In: Greer JP, Arbor DE, Glader B, et al., eds. Wintrobe's Clinical Hematology. 13th ed. Philadelphia: Wolters Kluwer, 2013.

4. Lawrie D, Coetzee LM, Becker P, Mahlangu J, Stevens W, Glencross DK. Local reference ranges for full blood count and CD4 lymphocyte count testing. S Afr Med J 2009;99(4):243-248.

5. Lopez A, Cacoub P, Macdougall IC, Peyrin-Biroulet L. Iron deficiency anaemia. Lancet 2016;387(10021):907916. http://dx.doi.org/10.1016/S0140-6736(15)60865-0

6. Punnonen K, Irjala K, Rajamaki A. Serum transferrin receptor and its ratio to serum ferritin in the diagnosis of iron deficiency. Blood 1997;89(3):1052-1057.

Erslev AJ. Erythropoietin and anemia of cancer. Eur J Haematol 2000;64(6):353-358. http://dx.doi Erslev AJ. Erythropoietin and anem
org/10.1034/j.1600-0609.2000.9r116.x

8. Henry DH, Beall GN, Benson CA, et al. Recombinant human erythropoietin in the treatment of anemia associated with human immunodeficiency virus (HIV) infection and zidovudine therapy. Overview of four clinical trials. Ann Intern Med 1992;117(9):739-748.

9. Auerbach $\mathrm{M}$, Ballard $\mathrm{H}$, Trout JR, et al. Intravenous iron optimizes the response to recombinant human erythropoietin in cancer patients with chemotherapy-related anemia: A multicenter, openlabel, randomized trial. J Clin Oncol 2004;22(7):1301-1307. http://dx.doi.org/10.1200/JCO.2004.08.119

10. Walters HM, Smith C, Howarth JE, et al. New enzyme immunoassay for detecting total, type I, and type II intrinsic factor antibodies. J Clin Pathol 1989;42(3):307-312. http://dx.doi.org/10.1136/jcp.42.3.307 